\title{
Resolution and Dose Dependence of Radiation Damage in Biomolecular Systems
}

\author{
Hakan Atakisia ${ }^{a}$ Lauren Conger ${ }^{a}$, David Moreau ${ }^{a}$, and Robert E. Thorne ${ }^{a}$ \\ aphysics Department, Cornell University, Ithaca, NY, 14853, USA, ha286@cornell.edu
}

Serial synchrotron-based crystallography using intense microfocused X-ray beams, fastframing detectors and protein microcrystals held at $300 \mathrm{~K}$ promises to expand the range of accessible structural targets and to increase overall structure-pipeline throughputs. To explore the nature and consequences of $\mathrm{X}$-ray radiation damage under microbeam illumination, we measured the time-, dose- and temperature-dependent evolution of crystal diffraction with maximum dose rates up to $\sim 50 \mathrm{MGy} \mathrm{s}^{-1}$. At all temperatures and dose rates the integrated diffraction intensity for a fixed crystal orientation shows nonexponential decays with dose. Non-exponential decays are a consequence of nonuniform illumination and the resulting spatial evolution of diffracted intensity within the illuminated crystal volume.

For Gaussian beams the diffraction weighted dose, which reflects the average damage state of the crystal regions contributing to diffraction at a given dose, becomes nearly independent of actual dose at large doses, as a result of "hole burning" by the intense beam centre. An apparent delayed onset of radiation damage seen in many intensitydose curves collected with fixed sample orientation is in fact a consequence of damageinduced redistribution of diffracted intensity in reciprocal space. Accounting for these effects, data collection at the highest dose rates increases crystal radiation lifetimes near $300 \mathrm{~K}$ (but not at $100 \mathrm{~K}$ ) by a factor of $1.5-2$ compared with those observed at conventional dose rates, for crystals that have ordinary room-temperature radiation sensitivities [1].

The fundamental relation of interest in understanding radiation damage in diffraction and imaging is the local Fourier-space relation between diffracted intensity $I$, diffraction wavevector $q$, and dose $D, \tilde{I}(q, D)$. Models used in protein crystallography for the last 50 years provide good fits to experimental $I(q)$ vs nominal dose data but have unclear physical significance. More recently, a fit to diffraction and imaging experiments suggested that the maximum tolerable dose varies as $q^{-1}$ or linearly with resolution. We have reanalyzed previous data for $I(q)$ versus dose to account for the effects spatially nonuniform crystal irradiation and diffraction during data collection. The reanalyzed data are consistent with a purely exponential local dose dependence, $\tilde{I}(q, D)=I_{0}(q) \exp \left(-D / D_{e}(q)\right)$, where $D_{e}(q) \propto q^{\alpha}$ with $\alpha \approx 1.7$. A physics-based model for radiation damage, in which damage events occurring at random locations within a sample each cause energy deposition and blurring of the electron density within a small volume, predicts this exponential variation with dose for all $q$ values, and a decay exponent $\alpha \approx 2$ in $2 \mathrm{D}$ and $3 \mathrm{D}$, roughly consistent with both diffraction and imaging experiments over more than two orders of magnitude in resolution [2]. This strong q/resolution dependence implies that the "dose limit" when collecting complete data sets cannot be meaningfully represented by a single number (e.g., the 30 MGy Garman limit), but in fact vary rapidly with desired maximum resolution and are less than $10 \mathrm{MGy}$ at $1.5 \AA$ resolution.

References

[1] Warkentin, M., et al., IUCrJ 4, 785-794 (2017).

[2] Atakisi, H., Conger, L, Moreau, D., and Thorne, R. E. submitted to IUCrJ 